\title{
Percepções ambientais e fatores associados à ocorrência de anticorpos anti-Leptospira sp. em cães de um reassentamento urbano no município de Porto Alegre, estado do Rio Grande do Sul, Brasil
}

\section{Environmental perceptions and factors associated with the occurrence of anti-Leptospira sp. in dogs of an urban resettlement in the City of Porto Alegre, Rio Grande do Sul State, Brazil}

Marilise Oliveira Mesquita', Graziella Chaves Trevilato', Michelle da Silva Schons', Luiza de Holleben Saraiva², Rogério Oliveira Rodrigues ${ }^{3}$, Luis Gustavo Corbellini²

' Universidade Federal do Rio Grande do Sul, Escola de Enfermagem, Porto Alegre, Rio Grande do Sul, Brasil

2 Universidade Federal do Rio Grande do Sul, Faculdade de Veterinária, Porto Alegre, Rio Grande do Sul, Brasil

${ }^{3}$ Instituto de Pesquisas Veterinárias Desidério Finamor, Laboratório de Leptospirose, Eldorado do Sul, Rio Grande do Sul, Brasil

\begin{abstract}
RESUMO
OBJETIVOS: Avaliar a frequência de soropositividade para Leptospira sp. nos cães de um grupo de famílias reassentadas e os fatores de risco para leptospirose canina (percepção dos moradores e fatores ambientais locais). MATERIAIS E MÉTODOS: O trabalho foi realizado em um reassentamento urbano, para o qual seus moradores foram transferidos de uma área de ocupação irregular (Vila Dique de Porto Alegre, Rio Grande do Sul), e com condições favoráveis à disseminação da leptospirose. Foi realizado um estudo transversal descritivo com entrevistas em 89 domicílios e 142 coletas de amostras de sangue de cães da comunidade, para sorologia antileptospira, no período de novembro de 2011 a dezembro de 2012. RESULTADOS: Das amostras analisadas, 18,3\% (26 cães) foram soropositivas, e a sorovariedade mais frequente foi a Icterohaemorrhagiae, presente em $46 \%$ das amostras. Para avaliar a hipótese de associação entre soropositividade para Leptospira sp. e percepções ambientais, cuidados com o ambiente e com os cães, foram realizados dois modelos de regressão logística para dados correlacionados. Apenas as variáveis "hábito de caçar ratos" e "acesso à rua" foram significativamente associadas à soropositividade $(p<0,05)$ no modelo univariado. $O$ modelo multivariado não resultou em diferença quando da inclusão dessas duas variáveis. CONCLUSÃO: Por meio das entrevistas e análises sanguíneas dos cães, foi possível verificar os riscos ambientais, no novo reassentamento, que contribuíram para a presença da leptospirose canina na comunidade.
\end{abstract}

Palavras-chave: Leptospirose; Fatores de Risco; Comunidades Vulneráveis; Zoonoses.

\begin{abstract}
OBJECTIVES: Evaluating the frequency of seropositivity to Leptospira sp. in dogs from a group of relocated families and risk factors for canine leptospirosis (local residents' perception and environmental factors). MATERIALS AND METHODS: The study was carried out in an urban resettlement, in which its inhabitants were transferred from an area of irregular occupation (Vila Dique in Porto Alegre, Rio Grande do Sul), and with favorable conditions to the spread of leptospirosis. A cross-sectional descriptive study was conducted with interviews in 89 households and 142 samples of blood collected from dogs in the community for anti-Leptospira serology, from November 2011 to December 2012. RESULTS: About the analyzed samples, 18.3\% (26 dogs) were seropositive, and the most frequent serovariety was Icterohaemorrhagiae which was present in $46 \%$ of the samples. In order to evaluate the hypothesis of association among seropositivity to Leptospira sp. and environmental perceptions, care for the environment and dogs, two logistic regression models were performed for correlated data. Only the variables "habit of hunting mice" and "access to the street" were significantly associated with seropositivity $(p<0.05)$ in the univariate model. The multivariate model did not show differences with these two variables. CONCLUSION: Through the interviews and blood tests of the dogs, it was possible to verify the environmental risks in the new resettlement, which contributed to the presence of canine leptospirosis in the community.
\end{abstract}

Keywords: Leptospirosis; Risk factors; Vulnerable Communities; Zoonoses.

\footnotetext{
Correspondência / Correspondence:

Marilise Oliveira Mesquita

Universidade Federal do Rio Grande do Sul, Escola de Enfermagem

Rua São Manoel, 963. Campus Saúde. Bairro: Rio Branco - CEP: 90620-1 10 - Porto Alegre, Rio Grande do Sul, Brasil

Tel.: +55 (51) 3308-5369

E-mail:marilise.mesquita@ufrgs.br
} 


\section{INTRODUÇÃO}

As comunidades que se organizam em ocupações irregulares nas grandes cidades são, muitas vezes, removidas para locais de menor interesse econômico, em função da exploração imobiliária e viária. Esse foi o caso da Vila Dique de Porto Alegre, no estado do Rio Grande do Sul, Brasil, que iniciou sua formação na década de 1960, com famílias oriundas de diversas partes do interior do Estado'. O local não tinha saneamento e apresentava polvição ambiental severa devido à deposição no ambiente de resíduos sólidos e esgoto doméstico ${ }^{2}$, com um número expressivo de moradores que eram catadores de materiais recicláveis ${ }^{3}$. $\bigcirc$ depósito inadequado dos resíduos sólidos domésticos em aglomerados urbanos constitui um ambiente favorável à atração de ratos (Rattus norvegicus), que trazem consigo microrganismos, como Leptospira sp., causadores de doenças. Esses resíduos, em condições de umidade, mantêm as leptospiras, eliminadas pela urina dos roedores, por mais tempo no ambiente ${ }^{3,4}$. Os animais domésticos, silvestres e sinantrópicos ${ }^{5,6}$ podem ser reservatórios e fontes dessa bactéria, sendo potenciais transmissores de doenças para os humanos ${ }^{7}$. No ambiente urbano, o cão tem implicações em saúde pública, pois o animal pode ser responsável pela transmissão de várias zoonoses, dentre elas a leptospirose ${ }^{8}$. O baixo grau de instrução das pessoas que habitam locais sem saneamento as torna vulneráveis à leptospirose, indicando a necessidade de educação ambiental e sanitária para que as comunidades possam ter autonomia no cuidado com

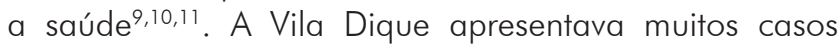
de leptospirose humana ${ }^{3}$. $\bigcirc$ objetivo do trabalho foi avaliar a frequência de soropositividade à Leptospira sp. nos cães dessa comunidade urbana reassentada, e os fatores de risco para leptospirose canina envolvendo a percepção de seus moradores sobre a transmissão da enfermidade e os cuidados sanitários com o ambiente e com os cães.

\section{MATERIAIS E MÉTODOS}

A pesquisa foi realizada em um novo reassentamento urbano, para o qual seus residentes foram transferidos de uma área periférica da cidade que fora ocupada irregularmente na década de 1960 (Vila Dique de Porto Alegre, estado do Rio Grande do Sul). O novo conjunto habitacional, com saneamento adequado, conta com infraestrutura urbana, como: central de triagem de materiais recicláveis, centro comunitário, creche, unidade básica de saúde e escola de ensino fundamental.

Trata-se de um estudo transversal desenvolvido por meio de entrevistas domiciliares e análises sorológicas nos cães da comunidade no período de novembro de 2011 a dezembro de 2012. Os moradores foram visitados e responderam a um questionário com 26 perguntas estruturadas sobre a presença de animais sinantrópicos dentro dos domicílios e medidas de controle; percepções quanto a possíveis zoonoses transmitidas pelos cães; perguntas sobre leptospirose humana, cuidados e posse responsável dos cães (animal com acesso à rua); presença de ectoparasitos; e segregação e acúmulo de resíduos sólidos domésticos. Os critérios de inclusão para responder ao questionário foram: possuir pelo menos um cão no domicílio e ser maior de 18 anos de idade. Todos os entrevistados assinaram um Termo de Consentimento Livre e Esclarecido, redigido em duas vias, permanecendo uma via com o entrevistado, conforme as normas expressas na Resolução CNS/MS n ${ }^{\circ} 466$ de $2012^{12}$. O projeto foi aprovado pelo Comitê de Ética em Pesquisa da Universidade Federal do Rio Grande do Sul sob o $n^{\circ} 20648$.

Análises sorológicas antileptospira foram realizadas nos cães dos 89 moradores entrevistados, tendo sido realizadas 142 coletas de sangue com a autorização do proprietário do animal. Os proprietários informaram que os animais não haviam recebido nenhum tipo de vacina previamente. As análises foram realizadas no Laboratório de Leptospirose do Instituto de Pesquisas Veterinárias Desidério Finamor (FEPAGRO Saúde Animal), localizado em Eldorado do Sul, estado do Rio Grande do Sul. A técnica utilizada pelo Laboratório foi a soroaglutinação microscópica, sendo utilizados no diagnóstico 18 sorovares de Leptospira interrogans: Australis, Autumnalis, Ballum, Canicola, Copenhageni, Celledoni, Cynopteri, Hardjo, Icterohaemorrhagiae, Javanica, Panama, Pyrogenes, Pomona, Tarassovi, Wolffi, Castelonis, Bataviae e Saxkoebing. A técnica utilizada determinou quantitativamente o título dos anticorpos para cada uma das sorovariedades da bactéria, sendo considerados positivos os animais que apresentaram títulos iguais ou superiores a 100, conforme proposto pela Organização Internacional para Saúde Animal, pela Organização Pan-Americana da Saúde e pela Organização Mundial da Saúde ${ }^{13}$. Foram avaliadas as frequências de animais soropositivos e as frequências das sorovariedades encontradas.

Para avaliar os fatores associados entre a soropositividade e a percepção da leptospirose e cuidados com os cães, foram realizados dois modelos de regressão logística para dados correlacionados. Como as coletas foram efetuadas no período de um ano, e a sazonalidade pode influenciar a ocorrência de leptospirose canina, uma estrutura de correlação entre as amostras, em cada data de coleta, foi incluída. Dois modelos de regressão foram construídos: 1) incluindo dados relativos ao animal (idade, sexo, porte), saúde (presença de ectoparasitos) e comportamento (hábito de caçar ratos e acesso à rua); e 2) incluindo dados sobre a presença de animais sinantrópicos nos domicílios, hábito de deixar restos de comida no prato do cão, costume de acumular materiais recicláveis em casa, segregação dos resíduos sólidos domésticos, conhecimento de alguém que já teve leptospirose e conhecimento de que o cão pode transmiti-la.

Inicialmente foi aplicado um modelo univariado incluindo as variáveis independentes acima citadas. Todas as variáveis com $p<0,20$ foram selecionadas 
para a construção de um modelo multivariado. $\bigcirc$ modelo foi construído pelo processo forward, sendo que somente as variáveis com $\mathrm{p}<0,05$ permaneceram no modelo final. $\bigcirc$ modelo mais ajustado foi selecionado pelo valor de QIC (critério de quase-verossimilhança). A variável idade dos cães foi testada como confundidor, e, se a inclusão da idade alterasse os estimadores das outras variáveis dependentes (> 20\%), essa seria mantida no modelo. Todas as variáveis pré-selecionadas foram testadas para correlação. As análises foram realizadas utilizando-se o pacote estatístico SAS e procedimento PROC GENMOD.

\section{RESULTADOS}

Dos animais analisados, 18,3\% (26) foram soropositivos para Leptospira sp. As frequências das sorovariedades encontradas nas amostras de sangue dos cães foram: Icterohaemorrhagiae (12); Canicola (sete); Pomona (quatro); Autumnalis (quatro), Copenhageni (três) e Pyrogenes (dois). No presente estudo, as titulações na prova de soroaglutinação variaram de 100 a 400. As titulações de 100 ocorreram em 25 reações $(80,6 \%)$, de 200 em cinco reações $(16,1 \%)$ e 400 em apenas uma reação $(3,2 \%)$.

$\mathrm{Na}$ primeira análise com as variáveis dos cães, como idade, sexo, porte e possíveis fatores de risco (acesso à rua, hábito de caçar ratos, presença de ectoparasitoses), apenas as variáveis "hábito de caçar ratos" e "acesso à rua" foram significativamente associadas à soropositividade $(p<0,05)$ no modelo univariado. $\bigcirc$ modelo multivariado não resultou em significância estatística quando da inclusão dessas duas variáveis. De acordo com o critério de ajuste dos modelos (menor QIC), a variável que mais explicou a soropositividade foi "hábito de caçar ratos"; a inclusão da idade não alterou substancialmente o estimador dessa variável. Os cães com o hábito de caçar ratos apresentaram uma chance maior de serem soropositivos comparados com os que não possuíam esse hábito (OR = 1,20; IC 95\%: 1.002-1.459).

Os resultados da segunda análise indicaram que apenas a segregação dos resíduos domiciliares foi mais próxima de estar associada à maior frequência de soropositividade ( $p=0,06$; estimate $=0,133$ ). Para as demais variáveis independentes, obteve-se um valor de p não significativo.

Com relação às zoonoses, foi questionado aos moradores se eles conheciam alguém da comunidade que tivesse sido acometido por leptospirose: $40 \%$ deles responderam afirmativamente. Quando foi perguntado se a pessoa sabia que o cão pode transmitir leptospirose, $71 \%$ responderam que não sabiam. Foi observado, nas respostas das entrevistas, que $100 \%$ das residências apresentavam pelo menos um tipo de animal sinantrópico, sendo que a maioria dos moradores convivia com mais de um tipo: $63 \%$ das casas tinham carrapatos; $61 \%$ tinham ratos; $50 \%$ tinham pombos; $47 \%$ tinham mosquitos; e 39\% das casas tinham pulgas. Com relação ao controle de animais sinantrópicos dentro dos domicílios, 27\% das residências não realizavam nenhum tipo de controle.

\section{DISCUSSÃO}

A frequência de soropositividade Leptospira sp. neste estudo foi de 18,3\% (26 cães). A alta prevalência de ratos nos domicílios (61\%) corrobora a frequência de soropositividade antileptospira nos cães. A frequência total de soropositivos se assemelha a vários estudos epidemiológicos realizados em outras regiões urbanas do país ${ }^{14,15,16}$. Contudo, o que varia entre os estudos são as sorovariedades mais incidentes. A sorovariedade Icterohaemorrhagiae apresentou uma frequência de $46 \%$ nas amostras de soro canino avaliadas no presente estudo, o que indica um risco à saúde pública, pelo fato de ser essa a sorovariedade responsável pelos quadros mais graves para as pessoas. A segunda sorovariedade mais prevalente foi a Canicola, com $27 \%$.

De acordo com vários estudos, as prevalências das sorovariedades mudam de acordo com os fatores ambientais, ecológicos, demográficos e geográficos de cada estudo $5,16,17,18,19,20$. Essas variações nos índices de soropositividade que ocorrem em diferentes ambientes fazem com que se confirme que a contaminação por Leptospira sp. está condicionada a uma grande diversidade de situações e fatores ambientais, tais como temperatura e pluviosidade ${ }^{21}$, reservatórios, altitude e bacias hidrográficas ${ }^{22}$, características da organização espacial, dos ecossistemas e das condições de vida e trabalho da população ${ }^{23}$.

No presente estudo, as titulações positivas na prova de soroaglutinação variaram de 100 a 400, sendo 100 (80,6\%), $200(16,1 \%)$ e 400 (3,2\%). Titulações de 100 e 200 são consideradas baixas e podem significar título residual de infecção prévia ou de infecção recéminstalada ${ }^{24}$. Por se tratar de uma comunidade de baixo poder econômico, os animais que tiveram amostras de sangue analisadas não haviam recebido nenhum tipo de vacina, portanto esses títulos foram produzidos pelo contato com a bactéria no ambiente. Destaca-se que nem todos os cães que tiveram amostras de sangue coletadas eram provenientes da antiga Vila Dique; alguns cães mais novos foram adquiridos no novo local. No entanto, a soropositividade para leptospira apresenta duração variável, sendo provável que esses cães soropositivos tenham entrado em contato com a leptospira no novo ambiente. Ainda deve ser ressaltado que existem reações cruzadas entre sorovariedades que se assemelham entre si, como é o caso da Icterohaemorrhagiae e a Pyrogenes, que são sorovariedades muito semelhantes em termos antigênicos. Por isso, os resultados da sorologia devem ser analisados com cautela ${ }^{25}$.

Cães que possuíam o hábito de caçar ratos e acesso à rua apresentaram chances maiores de serem soropositivos que os cães que não caçavam ratos. Esse resultado também foi encontrado em outro estudo $^{17}$, observando-se que os cães com acesso 
à rua apresentaram um risco 2,57 vezes maior de se infectarem em relação àqueles que não tiveram acesso à rua. Nesse mesmo estudo, foi encontrado um risco 4,22 vezes maior de serem soropositivos aqueles que possuíam o hábito de caçar roedores. Em outro estudo, Silva et al. ${ }^{26}$ constataram que cães com acesso à rua apresentaram títulos sorológicos mais altos, comparados aos animais domiciliados. $\bigcirc$ hábito de caçar ratos já foi identificado por Fraga ${ }^{19}$ como fator de risco para a infecção canina por leptospirose.

Os resultados da segunda análise de risco indicaram que a segregação dos resíduos domiciliares foi mais próxima de estar associada à maior frequência de soropositividade para leptospirose nos cães ( $p=0,06$; estimate $=0,133$ ). Para as demais variáveis independentes, obteve-se um valor de $p$ não significativo. A comunidade estudada apresentava um grande número de pessoas que se dedicavam à catação de resíduos sólidos ${ }^{3}$ e tinham por hábito acumular esses materiais recicláveis em sua área residencial. Essa prática atrai animais sinantrópicos e, com eles, uma maior probabilidade de contaminação dos cães por leptospirose. $\bigcirc$ fato de $61 \%$ das residências apresentarem ratos no seu interior acentua a possibilidade da contaminação dos cães também nessas residências ou em seus pátios. $\bigcirc$ mesmo foi descrito por Santos et al. ${ }^{27}$ numa comunidade de catadores na periferia de Brasília, Distrito Federal, em que $90 \%$ dos domicílios estudados tinham ratos e baratas.

As zoonoses, em especial a leptospirose, são enfermidades bastante frequentes em comunidades sem acesso ao saneamento básico ou em condições de vulnerabilidade ambiental e social ${ }^{28}$. Foi observado, pelas respostas das entrevistas, que $40 \%$ das pessoas conheciam alguém que havia sido acometido por leptospirose e 29\% dos respondentes reconheceram - cão como possível transmissor dessa doença. $\mathrm{Na}$ maioria dos casos, apenas o rato é reconhecido como o causador da leptospirose, enfermidade popularmente chamada como "doença do rato"3.

\section{CONCLUSÃO}

A frequência de soropositividade nos cães deste estudo não foi muito diferente da de outros trabalhos publicados em distintos contextos nas diversas partes do país, porém a maior prevalência da sorovariedade Icterohaemorrhagiae alerta para - risco à saúde das pessoas desta comunidade. As condições ambientais encontradas naquela população reassentada são favoráveis à disseminação da leptospirose, devido ao acúmulo de resíduos sólidos nas casas e alto índice de roedores nas residências. Ações de educação ambiental são necessárias em comunidades de áreas sem saneamento básico ou provenientes delas, na forma de educação não formal, introduzindo novos conhecimentos como meios de proteção à saúde dessas pessoas. $\bigcirc$ trabalho de educação e conscientização ambiental para as comunidades reassentadas pode ser um efetivo instrumento de prevenção de zoonoses, sendo essas atividades parte da promoção e vigilância em saúde.

\section{APOIO FINANCEIRO}

Os autores agradecem à Pró-Reitoria de Pesquisa e à Pró-Reitoria de Extensão da Universidade Federal do Rio Grande do Sul pelo apoio financeiro e pelas bolsas de pesquisa e extensão.

\section{REFERÊNCIAS}

1 Gil CZV. Da Vila Dique ao Porto Novo: extensão popular, rodas de memórias e remoções urbanas. São Leopoldo: Oikos; 2013.

2 Troleis AL. Metrópole de risco: o caso da Vila Dique e do aterro sanitário da zona norte na polvição das águas superficiais e subterrâneas das bacias hidrográficas dos Arroios da Areia e Passo das Pedras/Porto Alegre, RS [tese]. Porto Alegre (RS): Universidade Federal do Rio Grande do Sul, Programa de Pós-graduação em Geografia; 2009.

3 Zacarias IR, Bavaresco CS. Conhecendo a realidade dos catadores de materiais recicláveis da Vila Dique: visões sobre os processos de saúde e doença. Textos Contextos. 2009 jul-dez;8(2):293-305

4 Pahren HR, Clark CS. Microorganisms in municipal solid waste and public health implications. Crit Rev Environ Control. 1987;17(3):187-228.
5 Silva FJ, Mathias LA, Magajevski FS, Werther K, Assis NA, Girio RJS. Anticorpos contra Leptospira spp. em animais domésticos e silvestres presentes no campus universitário da FCAV, UNESP, Jaboticabal/SP. Ars Vet. 2010 jul;26(1):17-25.

6 Silva FJ, Santos CEP, Silva GCP, Santos RF, Curci VCM, Mathias LA. The importance of Leptospira interrogans serovars Icterohaemorrhagiae and Canicola in coastal zone and in southern fields of Rio Grande do Sul, Brazil. Pesq Vet Bras. 2014 Jan;34(1):34-8.

7 Genovez ME. Leptospirose: uma doença de ocorrência além da época das chuvas! Biologico. 2009 jan-jun;71 (1): 1 -3.

8 Batista CSA, Azevedo SS, Alves CJ, Vasconcellos SA, Morais ZM, Clementino IJ, et al. Soroprevalência de leptospirose em cães errantes da cidade de Patos, Estado da Paraíba, Brasil. Braz J Vet Res Anim Sci. 2004 mar-abr;41 (2):131-6. 
9 Dias GF. Educação ambiental: princípios e práticas. 9. ed. São Paulo: Gaia; 2010.

10 Lermen HS, Fisher PD. Percepção ambiental como fator de saúde pública em área de vulnerabilidade social no Brasil. Rev APS. 2010 jan-mar; 13(1):62-71.

11 Mesquita MO, Trevilato GC, Saraiva LH, Schons MS, Garcia MIF. Material de educação ambiental como estratégia de prevenção da leptospirose para uma comunidade urbana reassentada. Cad Saude Colet. 2016 jan-mar;24(1):77-83.

12 Brasil. Ministério da Saúde. Conselho Nacional de Saúde. Resolução n 466, de 12 de dezembro de 2012. Diretrizes e normas regulamentadoras de pesquisa envolvendo seres humanos. Diário Oficial da União, Brasília (DF), 2013 jun 13; Seção 1:59.

13 World Health Organization; Regional Medical Research Centre. Indian Council of Medical Research. Leptospirosis: laboratory manual. New Delhi: Indian Council of Medical Research; 2007.

14 Lilenbaum W, Rodrigues F, Barboza F. Aglutininas antileptospiras em caninos do município amazônico de Oriximiná-Pará, Brasil. Rev Bras Cienc Vet. 2000 set-dez;7(3): 133-5.

15 Aguiar DM, Cavalcante GT, Marvulo MFV, Silva JCR, Pinter A, Vasconcellos SA, et al. Fatores de risco associados à ocorrência de anticorpos antiLeptospira spp. em cães do município de Monte Negro, Rondônia, Amazônia Ocidental Brasileira. Arq Bras Med Vet Zootec. 2007 fev;59(1):70-6.

16 Silva WB, Simões LB, Padovani CR, Langoni $H$, Lopes ALS, Modolo JR. Inquérito sorológico e distribuição espacial da leptospirose canina em área territorial urbana da cidade de Botucatu, São Paulo. Vet Zootec. 2009 dez; 16(4):656-68.

17 Querino AMV, Delbem ACB, Oliveira RC, Silva FG, Müller EE, Freire RL, et al. Fatores de risco associados à leptospirose em cães do município de Londrina-PR. Semin Cienc Agrar. 2003 jan-jun;24(1):27-34.

18 Rodríguez $A L$, Ferro $B E$, Varona $M X$, Santafé M. Evidencia de exposición a Leptospira en perros callejeros de Cali. Biomedica. 2004 sep;24(3):291-5.

19 Fraga DBM. Avaliação do papel do cão como reservatório na transmissão da leptospirose urbana [tese]. Salvador (BA): Fundação Oswaldo Cruz, Centro de Pesquisas Gonçalo Moniz; 2008. 78 p.
20 Jouglard SDD, Brod CS. Leptospirose em cães: prevalência e fatores de risco no meio rural do Município de Pelotas, RS. Arq Inst Biol. 2000 jul-dez;67(2): 181-5.

21 Mesquita M, Pellegrini DCP, Simões Pires Neto JA, Reis GR, Medeiros C, Corbellini LG. Análise de série temporal para avaliação do perfil sorológico da leptospirose bovina no Estado do Rio Grande do Sul de 1996 a 2006. Arq Inst Biol. 2010 jul-set;77(3):381-7.

22 Barcellos C, Lammerhirt CB, Almeida MAB, Santos E. Distribuição espacial da leptospirose no Rio Grande do Sul, Brasil: recuperando a ecologia dos estudos ecológicos. Cad Saude Publica. 2003 set-out; 19(5): 1283-92.

23 Murhekar MV, Sugunan AP, Vijayachari P, Sharma S, Sehgal SC. Risk factors in the transmission of leptospiral infection. Indian J Med Res. 1998 May; 107:218-23.

24 Blazius RD, Romão PRT, Blazius EMCG, Silva OS. Ocorrência de cães errantes soropositivos para Leptospira spp. na Cidade de Itapema, Santa Catarina, Brasil. Cad Saude Publica. 2005 nov-dez;21 (6):1952-6.

25 Faine S, Adler B, Bolin C, Perolat P. Leptospira and leptospirosis. 2nd ed. Melbourne: MedSci; 1999. 272 p.

26 Silva WB, Simões LB, Lopes ALS, Padovani CR, Langoni $H$, Modolo JR. Avaliação de fatores de risco de cães sororreagentes à Leptospira spp. e sua distribuição espacial, em área territorial urbana. Braz J Vet Res Anim Sci. 2006;43(6):783-92.

27 Santos LMP, Carneiro FF, Hoefel MGL, Santos W, Nogueira TQ. The precarious livelihood in waste dumps: a report on food insecurity and hunger among recyclable waste collectors. Rev Nutr. 2013 May-Jun;26(3):323-34.

28 Figueiredo CM, Mourão AC, Oliveira MAA, Alves WR, Ooteman MC, Chamone CB, et al. Leptospirose humana no município de Belo Horizonte, Minas Gerais, Brasil: uma abordagem geográfica. Rev Soc Bras Med Trop. 2001 jul-ago;34(4):331-8. 